\title{
Rectal Duplication Cyst in a 12 year old Female Presenting with Chronic Constipation and Rectal Bleeding: A Case Report
}

\author{
Kimberly Harris ${ }^{1}$, Kishore Vellody ${ }^{2}$ \\ ${ }^{1}$ University of Pittsburgh Medical Center, UPMC Montefiore Hospital, Pittsburgh, USA; ${ }^{2}$ University of Pittsburgh School of Medi- \\ cine, Children’s Hospital of Pittsburgh, Pittsburgh, USA. \\ Email: harriska@upmc.edu, kishore.vellody@chp.edu
}

Received October 14 ${ }^{\text {th }}$, 2010; revised December 25 ${ }^{\text {th }}$, 2010; accepted December $29^{\text {th }}, 2010$.

\begin{abstract}
Constipation is a common presenting complaint in children. Rectal duplication cysts are rare congenital malformations that need to be considered in patients with chronic constipation that has not responded to typical therapy and in patients presenting with rectal bleeding. It is important to maintain a high index of suspicion when diagnosing this condition as other congenital malformations may be present and rectal duplication cysts have the potential for malignant transformation if they go unrecognized. This case report describes a 12 year old female with chronic constipation and rectal bleeding who was found to have a rectal duplication cyst. It discusses the pathophysiology of the disease and highlights the options available for treatment.
\end{abstract}

Keywords: Rectal Duplication Cyst, Rectal Bleeding, Constipation, Rectal Adenocarcinoma

\section{Introduction}

Constipation is a common presenting complaint in children, accounting for up to $5 \%$ of all pediatrician appointments and an additional \$3.9 billion per year in healthcare costs compared to children without constipation [1]. Although most cases fall into the category of functional constipation, a small percentage of children have true organic causes of their symptoms. If a patient is not responding to traditional therapies for constipation, then other diagnoses need to be considered. One rare, but potentially serious cause of chronic constipation is a rectal duplication cyst.

Alimentary tract duplications are rare congenital malformations seen in 1 out of every 4500 autopsies. The most common location of an alimentary duplication is within the small intestine, comprising $44 \%$ of all cases, followed by the colon at $15 \%$ of all cases. Rectal duplications represent approximately $5 \%$ of alimentary tract duplications [2] with fewer than 100 reported cases in the literature. While some remain asymptomatic throughout a person's life, others can cause a wide variety of complications including hemorrhage, sepsis, and the potential for malignant transformation. Delayed diagnosis increases the risk of these complications; there- fore, it is important to maintain a high index of suspicion in cases where this diagnosis may be considered. In this report, we will present the case of a 12-year-old female with chronic constipation and rectal bleeding who was found to have a rectal duplication cyst and developed a complicated clinical course due to delayed diagnosis. We will review the clinical and radiological tools used in early detection of rectal duplication cysts, discuss the diagnostic implications of this congenital malformation, and note the management options that are currently available.

\section{Case Report}

A 12-year-old female presented to the emergency department with rectal bleeding. She reported a ten year history of constipation requiring the use of fiber supplements and laxatives in order to have regular bowel movements. On the day of presentation, the patient described passing a large non-bloody bowel movement with associated dyschezia and sacral numbness following several days of constipation and medication noncompliance. Shortly afterwards, she developed painless rectal bleeding and was brought to the emergency department for evaluation. 
Review of systems was negative for fever, vomiting, or diarrhea and she had not yet started her menses. She denied previous melena or hematochezia. Her past medical history was unremarkable, and there was no family history of gastrointestinal inflammatory diseases or cancer. She had never been evaluated by a gastroenterologist for constipation. Physical examination demonstrated a benign abdomen. Digital rectal examination revealed a moderate amount of bright red blood and clots. The exam was limited due to significant patient discomfort and tenderness, but no abnormalities were palpated. The initial hemoglobin was $8.5 \mathrm{gm} / \mathrm{dL}$ (normal 12-16 gm/dL) with normal indices and a reticulocyte count of $1.2 \%$ (normal 0.4-2.5\%) indicated likely brisk, acute blood loss.

Initial imaging with a technetium scan was negative for heterotopic gastric mucosa that would be consistent with a Meckel diverticulum. Esophagogastroduodenoscopy and colonoscopy revealed a normal appearing stomach and duodenum with dark blood throughout the colon, but no source of active bleeding. Computed tomography (CT) of the patient's abdomen and pelvis showed a $5.8 \times 5.4 \times 6.0 \mathrm{~cm}$ heterogeneous fluid collection posterior to the anal canal and displacing the lumen anteriorly (Figure 1). A pelvic ultrasound also showed the mass, but did not provide any additional information. A barium enema confirmed the mass which was displacing the rectum anteriorly, but failed to show any communication between the two structures. Magnetic resonance imaging (MRI) for better visualization revealed a well-circumscribed, low-attenuating rim around the hematoma (Figures 2(a) and 2(b)), which was felt to represent a component of the rectal wall. After reviewing the patient's his- tory and clinical findings, it was felt that the most likely diagnosis was that of a rectal duplication cyst, and

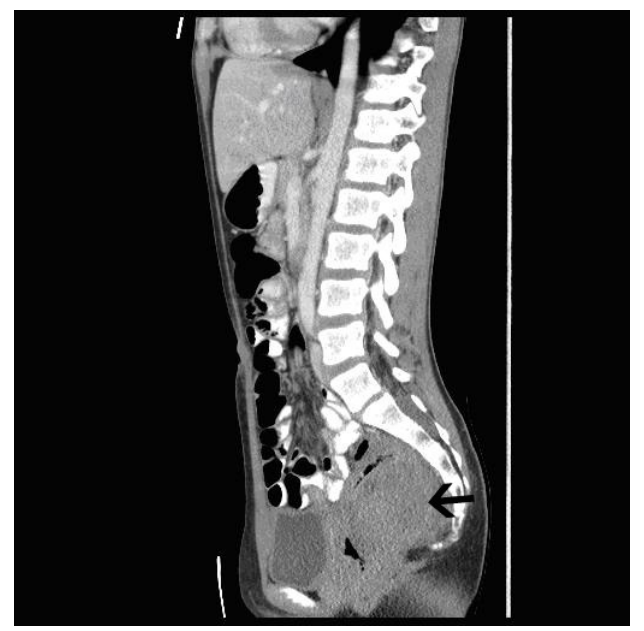

Figure 1. Midsagittal CT image showing the mass posterior to the anal canal (arrow) and displacing the rectal lumen anteriorly.

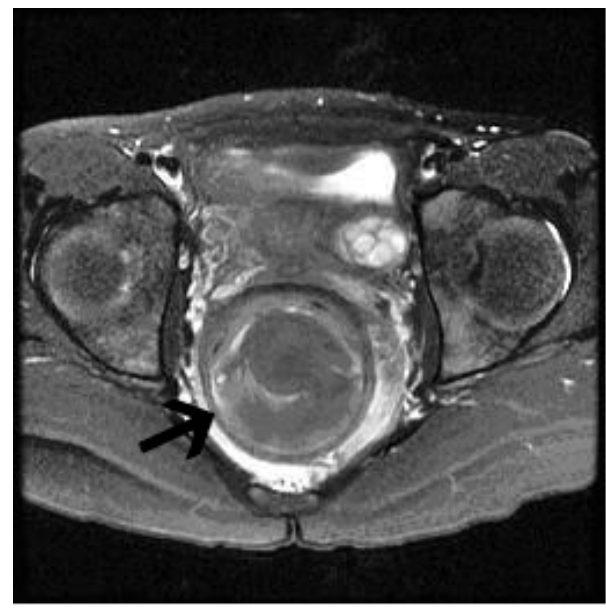

(a)

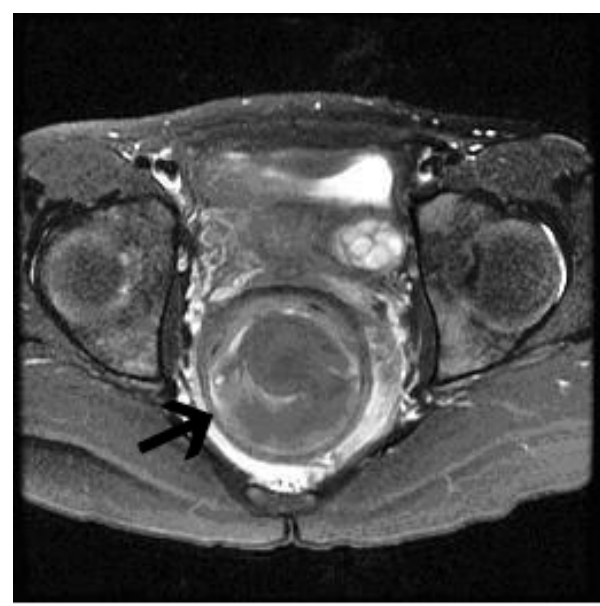

(b)

Figure 2. T2 transverse (a) and midsagittal (b) MRI images showing the fluid-filled cyst (arrow) surrounded by a well-circumscribed, low-attenuating muscular layer.

the patient was taken to the operating room for further evaluation and intervention.

Under anesthesia, a flexible sigmoidoscopy demonstrated a communication to the rectum just proximal to the anal sphincter. The opening was surrounded by normal appearing mucosa, while the cavity was lined by ulcerated, inflamed mucosa. There was no proximal communication with the rectum, and the bimanual exam revealed a wall between the posterior surface of the rectum and the anterior surface of the cyst consistent with a longitudinal rectal duplication cyst with a common wall. The cyst was partial excised via a posterior sagittal approach. The entire cyst could not be excised due to its intimate proximity with the anal sphincter. Histopathology showed hemorrhagic ulcerated colonic mucosa with mural fragment and granulation tissue surrounded by two thick muscle layers, confirming the diagnosis of a rectal duplication cyst. 
The patient's clinical course was complicated by the need for multiple blood transfusions. On post-operative day 10 , she returned to the hospital with fever and pain. A repeat CT showed a fluid collection in the presacral space and she was taken back to the operating room for drain placement. Three days later, she returned to the operating room after developing sepsis and drainage from her wound site. She was found to have a rectal perforation necessitating creation of a sigmoid colostomy and Hartmann pouch. The colostomy was closed 7 months later and she now has one formed bowel movement each day with full continence.

\section{Discussion}

Constipation is an exceedingly common issue, accounting for nearly $25 \%$ of consults to pediatric gastroenterologists. Fortunately, only a small minority of children have an organic cause to their constipation. It is important to uncover details regarding a delay in the passage of meconium, failure to thrive, blood in stools, abdominal distention, bilious vomiting, abnormal neurologic findings, or recurrent infections to differentiate functional constipation from more serious medical issues like Hirschsprung disease, hypothyroidism, spinal cord abnormalities, or cystic fibrosis [3]. In the patient presented, the predominantly concerning historical finding was the duration and severity of her constipation over 10 years of her life despite dietary changes and laxative use. Her rectal bleeding and suspected acute blood loss leading to anemia also made a pathologic etiology much more common. This led to gastrointestinal and surgical consultation and eventual discovery of the rectal duplication cyst.

Although the exact etiology of rectal duplication cysts is not known, several theories have been proposed. A split notochord [4] may explain some, but not all duplications found. Others may occur as the result of "partial twinning" and are usually associated with duplications of other structures within the genital and urinary tract. Persistent embryological diverticula or aberrant luminal recanalization [5] have also been proposed as possible etiologies. Finally, more rare causes include intrauterine trauma or hypoxia. Based on their embryogenesis, rectal duplication cysts are almost always located posterior to the rectum, with only four reported cases of anterior rectal duplication cysts [6].

While most rectal duplication cysts can be seen with contrast imaging, true diagnosis is rarely made before surgery. In 1940, Ladd and Gross proposed three essential characteristics that must be present to confirm the diagnosis of an alimentary tract duplication [7]: (1) a well-developed smooth muscle coat; (2) mucosal lining found within some portion of the alimentary tract; (3) contiguity to any segment of the alimentary tract. If the muscular layer is missing, then the malformation is classified as a tailgut cyst [2].

Rectal duplication cysts have a bimodal presentation with most being discovered either during the perinatal period or during early childhood. Up to $45 \%$ of all rectal duplication cysts are associated with a posterior midline fistula to the anus or perianal region that can be seen on physical exam. If a fistula is noted, further evaluation with an injected contrast study may confirm the presence of a rectal duplication cyst. Duplications without associated fistulas may be more difficult to diagnose. As the child ages, he or she can develop symptoms related to the cyst, including chronic constipation, rectal bleeding, rectal prolapse, hemorrhoids, and perirectal abscess formation. Any child that does not respond to traditional treatment for constipation or who develops any of the above complications should undergo further evaluation. An abdominal ultrasound is a reasonable first-pass study due to ease and lack of radiation exposure, but further imaging may be required if an abnormality is noted.

Other congenital genitourinary and vertebral abnormalities may be present in patients with rectal duplication cysts. Ravitch [8] and Yousefzadeh [9] reported such malformations including double bladder, double external genitals, double uterus or vagina, hemivertebra, separation of the pubis symphysis and cardiac abnormalities. While the patient discussed in this case report did not have any of these associated abnormalities, presence of one or more of these malformations should prompt further evaluation for a potential rectal duplication cyst.

The treatment of choice for rectal duplication cysts is surgical excision. The general approach involves surgical exposure, incision and drainage of the cystic fluid and stripping of the cystic mucosa to prevent future ulceration of gastric mucosa, if present. Although it is not always required since the rectum and cyst usually share a muscular wall, total excision may be performed via the posterior sagittal approach as used in our patient.

Timely diagnosis is important to prevent long-term complications of rectal duplication cysts. Ballantyne [10] reported on the first neoplastic transformation of an untreated rectal duplication cyst in 1932. Malignant degeneration occurs in between $7-18 \%$ of adult cases, with adenocarcinoma being the most commonly found malignancy. To date, no malignant transformation of a rectal duplication cyst has been reported in the pediatric population.

\section{Conclusion}

Rectal duplication cysts are a rare, but serious cause of chronic constipation and rectal bleeding. They may be accompanied by other congenital malformations and have the potential for malignant degeneration if not di- 
agnosed in a timely fashion. It is important to maintain a high index of suspicion when this diagnosis is part of the differential. Timely diagnosis and surgical excision are essential to prevent acute issues like gastrointestinal blood loss and future issues like malignant degeneration to adenocarcinoma.

\section{REFERENCES}

[1] O. Liem, J. Harman, M. Benninga, et al., "Health Utilization and Cost Impact of Childhood Constipation in the United States," Journal of Pediatrics, Vol. 154, No. 2, 2009, pp. 258-262. doi:10.1016/j.jpeds.2008.07.060

[2] M. Kocaoglu and D. P. Frush, "Pediatric Presacral Masses,” RadioGraphics, Vol. 21, 2001, pp. 575-584.

[3] S. Baker, C. DiLorenzo, G. Liptak, et al., "Evaluation and Treatment of Constipation in Infants and Children: Recommendations of the North American Society for Pediatric Gastroenterology," Journal of Pediatric Gastroenterology and Nutrition, Vol. 43, No. 3, 2006, pp. e1-e13.

[4] J. F. R. Bentley and J. R. Smith, "Developmental Posterior Enteric Remnants and Spinal Malformations,” Archives of Disease in Childhood, Vol. 35, No. 2, 1960, pp. 76-86. doi:10.1136/adc.35.179.76
[5] J. L. Bremer, "Diverticula and Duplications of the Intestinal Tract," Archives of Pathology and Laboratory Medicine, Vol. 38, 1944, pp. 132-140.

[6] K. Amjadi, D. Poenaru, D. Soboleski, et al., "Anterior Rectal Duplication: A Diagnostic Challenge,” Journal of Pediatric Surgery, Vol. 35, No. 4, 2000, pp. 613-614. doi:10.1053/jpsu.2000.0350613

[7] W. E. Ladd and R. E. Gross, "Surgical Treatment of Duplication of the Alimentary Tract: Enterogenous Cysts, Enteric Cysts, or Ileum Duplex,” Surgery, Gynecology \& Obstetrics, Vol. 70, 1940, pp. 295-307.

[8] M. M. Ravitch, "Hind Gut Duplication-Doubling of Colon and of the Genital and Lower Urinary Tracts," Annals of Surgery, Vol. 137, No. 5, 1953, pp. 588-601. doi:10.1097/00000658-195305000-00002

[9] D. K. Yousefzadeh, G. H. Bleckers, J. H. Jackson Jr, et al., "Tubular Colonic Duplications-Review of 1876-1981 Literature,” Pediatric Radiology, Vol. 13, No. 2, 1983, pp. 65-71. doi:10.1007/BF02390103

[10] E. W. Ballantyne, "Sacrococcygeal Tumors: Adenocarcinoma of a Cystic Congenital Embryonal Remnant," Archives of Pathology and Laboratory Medicine, Vol. 14, 1932, pp. 1-9. 